\title{
Результаты хирургического лечения
}

\section{медиастинальных нейрогенных опухолей} типа «гантели»

\author{
Подобед А.В., Зарецкий С.В., Малькевич В.Т.
}

Государственное учреждение «Республиканский научно-практический центр онкологии и медицинской радиологии им. Н.Н. Александрова», 223040, агрогородок Лесной, Минский район, Республика Беларусь

\begin{abstract}
Актуальность. Медиастинальные опухоли типа «гантели» встречаются редко, они могут приводить к компрессии спинного мозга или структур средостения. На сегодняшний день к лечению этой патологии единого подхода нет.

Цель нашего исследования - улучшить результаты лечения медиастинальных нейрогенных опухолей типа «гантели» путем анализа собственного опыта хирургических вмешательств с последующей разработкой лечебного подхода для этого типа новообразований.

Материал и методы. Проведен ретроспективный анализ историй пациентов, перенесших операцию в нашем центре по поводу нейрогенных опухолей средостения типа «гантели» с января 2003 г. по декабрь 2019 г. Опухоль удаляли и с использованием стандартного подхода (сочетанная ламинэктомия с торако- или цервикостернотомией), и с применением минимально-инвазивных методик (сочетание гемиламинэктомии с торакоскопией или надключичным доступом). Изучены непосредственные и отдаленные результаты операций.

Результаты. В исследование были включены 14 пациентов, что составило 12,9\% всех нейрогенных опухолей средостения. По сравнению с торако- и цервикостернотомией сочетание ламинэктомии с видеоассистированной торакоскопией или надключичным доступом сопровождалось меньшими болевым синдромом, интраоперационной кровопотерей $(p=0,013)$, частотой осложнений $(p=0,008)$, длительностью дренирования плевральной полости $(p=0,015)$ и госпитализации $(p=0,005)$. Послеоперационной летальности не было. Медиана наблюдения составила $68,6 \pm 37,2$ мес. За это время ни у одного пациента не выявлено признаков локального рецидива.

Заключение. При нейрогенных опухолях типа «гантели» в качестве хирургического доступа целесообразно сочетание гемиламинэктомии с видеоассистированной торакоскопией или надключичным доступом (при локализации в верхней апертуре). Эта стратегия характеризуется малой травматичностью, позволяет удалить опухоль единым блоком и избежать потенциальной опасности рецидива.
\end{abstract}

Финансирование. Исследование не имело спонсорской поддержки.

Конфликт интересов. Авторы заявляют об отсутствии конфликта интересов.

Для цитирования: Подобед А.В., Зарецкий С.В., Малькевич В.Т. Результаты хирургического лечения медиастинальных нейрогенных опухолей типа «гантели» // Клиническая и экспериментальная хирургия. Журнал имени академика Б.В. Петровского. 2021. T. 9, № 1. C. 23-28. DOI: https://doi.org/10.33029/2308-1198-2021-9-1-23-28

Статья поступила в редакцию 03.09.2020. Принята в печать 29.01.2021.

Outcomes of minimally invasive surgery for treatment of mediastinal neurogenic dumbbell tumors

Podobed A.V., Zaretski S.V., Malkevich V.T.

N.N. Alexandrov National Cancer Center of Belarus, 223040, Lesnoy, Minsk Region, Republic of Belarus

Background. Mediastinal dumbbell tumors are rare and can compress the spinal cord or mediastinal structures. There is no single approach to the treatment of this pathology.
ДЛЯ КОРРЕСПОНДЕНЦИИ Подобед Александр Владимирович врач - торакальный хирург онкологического торакального отделения «РНПЦ ОМР им. Н.Н. Александрова» (агрогородок Лесной, Минский район, Республика Беларусь) E-mail: a_podobed@icloud.com https://orcid.org/0000-0003-05082765

Ключевые слова: опухоли типа «гантели», ламинэктомия, опухоль средостения, нейрогенные опухоли, торакоскопия
CORRESPONDENCE Alexandr V. Podobed - Thoracic Surgeon of Department of Thoracic Surgery, N.N. Alexandrov National Cancer Center of Belarus (Lesnoy, Minsk Region, Belarus) E-mail: a_podobed@icloud.com https://orcid.org/0000-0003-05082765 
Keywords: The aim of our study was to improve the results of treatment of mediastinal neurogenic dumbbell dumbbell tumors, laminectomy, mediastinum tumor, neurogenic tumors, thoracoscopy tumors by analyzing our own experience of surgical interventions with development of an optimal approach for such tumors.

Material and methods. A retrospective analysis was performed on 14 patients who underwent surgery for thoracic dumbbell tumors at our department during the period 2003 to 2019. Removal of the tumor was performed both using the standard approach - a combination of laminectomy with thoracotomy or cervicosternotomy, and using minimally invasive techniques - a combination of hemilaminectomy with thoracoscopy or supraclavicular access. The immediate and long-term results of operations have been studied.

Results. The study included 14 patients, which accounted for $12.9 \%$ of all neurogenic tumors of the mediastinum. In comparison with thoracotomy and cervicosternotomy, the combination of laminectomy with VATS or supraclavicular approach was accompanied by a decrease in pain syndrome, intraoperative blood loss $(p=0.013)$, complications $(p=0.008)$, duration of pleural drainage $(p=0.015)$ and hospitalization $(p=0.005)$. There was no postoperative mortality. At a mean follow-up of 68.6 months no patients showed recurrence of the tumor.

Conclusion. For neurogenic dumbbell tumors a combination of hemilaminectomy with VATS or supraclavicular access (if localized in the thoracic inlet) is advisable as a surgical approach, which are characterized by low trauma and allow the tumor to be removed in a single block and to avoid the potential danger of recurrence.

Funding. The study had no sponsor support.

Conflict of interests. The authors declare no conflict of interests.

For citation: Podobed A.V., Zaretski S.V., Malkevich V.T. Outcomes of minimally invasive surgery for treatment of mediastinal neurogenic dumbbell tumors. Clinical and Experimental Surgery. Petrovsky Journal. 2021; 9 (1): 23-8. D0I: https://doi. org/10.33029/2308-1198-2021-9-1-23-28 (in Russian)

Received 03.09.2020. Accepted 29.01.2021.

$\mathrm{H}$ ейрогенные опухоли составляют 20\% всех новообразований средостения у взрослых [1]. Особую сложность вызывают образования проникающие в спинномозговой канал, которые могут вызвать компрессию спинного мозга, так называемые опухоли типа «песочные часы» или «гантели» (англ. dumbbell, sand-glass или hour-glass tumors). Это особые варианты новообразований периферических нервов, состоящие из двух частей: внутриканальной и паравертебральной, соединенной перешейком в межпозвонковом отверстии. Новообразования типа «гантели» встречаются в 10\% нейрогенных медиастинальных опухолей [2].

На сегодняшний день остаются нерешенными вопросы выбора хирургического доступа и роли видеоассистированной торакоскопии (ВАТC) при лечении этой патологии. Еще более сложная проблема - выбор доступа при опухолях верхней апертуры (шейно-медиастинальных), проникающих в спинномозговой канал.

Цель нашего исследования - улучшить результаты лечения медиастинальных нейрогенных опухолей типа «гантели» путем анализа собственного опыта хирургических вмешательств с последующей разработкой лечебного подхода для этого типа опухолей.

\section{Материал и методы}

Проведен ретроспективный анализ данных пациентов с медиастинальными опухолями типа «гантели», которым проведено хирургическое вмешательство в онкологическом торакальном отделении РНПЦ онкологии и медицинской радиологии им. Н.Н. Александрова с января 2003 г. по декабрь 2019 г. Из исследования исключены пациенты с редкими гистологическими формами опухолей типа «гантели» (гемангиома, фибросаркома и др.). Всего в исследование включены 14 пациентов, что составило 12,9\% всех нейрогенных опухолей средостения.

Всем пациентам проведено комплексное обследование, включая компьютерную (КТ) и магнитно-резонансную томографию (МРТ) (рис. 1, 2). Хирургический доступ подбирали индивидуально, с учетом локализации опухоли, согласно данным лучевых методов исследования.

Операция выполнялась мультидисциплинарной бригадой вместе с нейрохирургами. На первом этапе выполняли ламинэктомию (гемиламинэктомию) с мобилизацией интраканального компонента опухоли и пересечением нервного корешка (рис. 3). Мобилизацию внутригрудного компонента и удаление опухоли осуществляли посредством BATC или торакотомии. 
При паравертебральных опухолях с проникновением в межпозвонковое отверстие и без отдельно лежащего интраканального узла удаление производилось посредством ВАТС.

Выбор хирургического доступа при апикальных (цервико-медиастинальных) опухолях осуществлялся в зависимости от размера опухоли, ее локализации, связи с магистральными сосудами и окружающими структурами. Как правило, при небольших опухолях (до 5 см) мобилизация с расположением более $50 \%$ ее объема в плевральной полости (средостении), удаление медиастинальной части проводились посредством BATC.

При локализации более 50\% опухоли на шее операцию начинали с надключичного доступа. Этот доступ малотравматичен, обеспечивает прекрасный контроль магистральных сосудов и плечевого нервного сплетения; в случае необходимости при крупных опухолях разрез продляли на грудину с последующим выполнением частичной стернотомии или дополняли ВАТС при трудности мобилизации внутригрудного компонента (рис. 4).

Статистический анализ выполняли с использованием программы IBM SPSS Statistics v.26 (разработчик - IBM Corporation). Количественные значения выражали в виде медианы (Ме) и квартилей [25; 75]. Количественные характеристики между группами сравнивали с помощью U-критерия Манна-Уитни, для оценки корреляции использовали коэффициент Спирмена. Различия считали статистически значимыми при $p<0,05$.

\section{Результаты}

По нашим данным, частота встречаемости этих новообразований составила $12,9 \%$ всех нейроген-

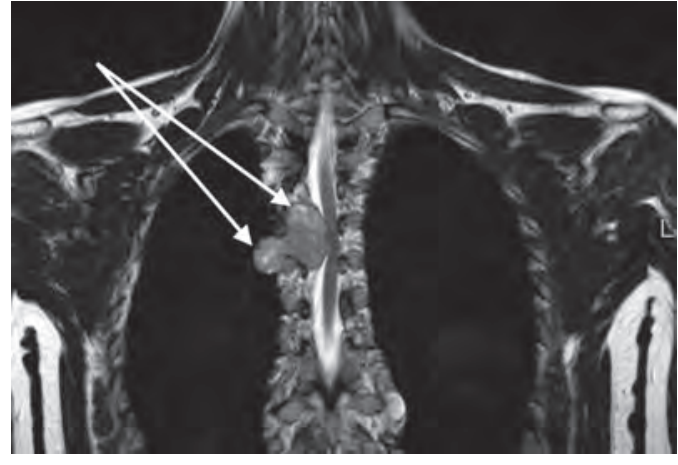

Рис. 1. Магнитнорезонансная томограмма пациента с опухолью по типу «гантели» (указана стрелками), фронтальный срез

Fig. 1. MRI of patient with dumbbell tumor (arrow)
Таблица 1. Характеристика пациентов с нейрогенными опухолями средостения

\begin{tabular}{|c|c|}
\hline Характеристика & Значение \\
\hline Возраст, годы & $35,9 \pm 19,0$ \\
\hline $\begin{array}{c}\text { Пол, } n(\%): \\
\text { мужчины } \\
\text { женщины }\end{array}$ & $\begin{array}{l}6(42,9) \\
8(57,1)\end{array}$ \\
\hline Размер опухоли, мм & $63,0 \pm 38,4$ \\
\hline ИМТ, $\mathrm{Kг} / \mathrm{M}^{2}$ & $25,7 \pm 5,9$ \\
\hline $0 Ф \mathrm{~B}_{1}, \%$ & $96,4 \pm 19,0$ \\
\hline $\begin{array}{l}\text { Жалобы, } n(\%): \\
\text { есть } \\
\text { нет }\end{array}$ & $\begin{array}{l}5(35,7) \\
9(64,3) \\
\end{array}$ \\
\hline $\begin{array}{c}\text { Сторона: } \\
\text { справа } \\
\text { слева }\end{array}$ & $\begin{array}{l}6(42,9 \%) \\
8(57,1 \%)\end{array}$ \\
\hline
\end{tabular}

ИМТ - индекс массы тела; 0ФВ выдоха за $1 \mathrm{c}$.

Таблица 2. Гистологические типы опухолей

\begin{tabular}{|l|c|}
\hline \multicolumn{1}{|c|}{ Гистология } & $\boldsymbol{n}(\%)$ \\
\hline Шваннома & $5(35,7)$ \\
\hline Ганглионеврома & $4(28,6)$ \\
\hline Нейрофиброма & $4(28,6)$ \\
\hline Злокачественная шваннома & $1(7,1)$ \\
\hline Всего & $14(100)$ \\
\hline
\end{tabular}

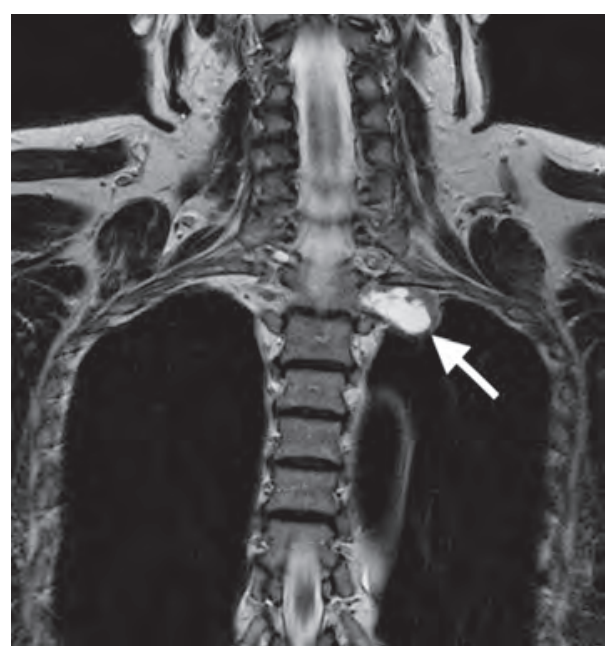

A (A)

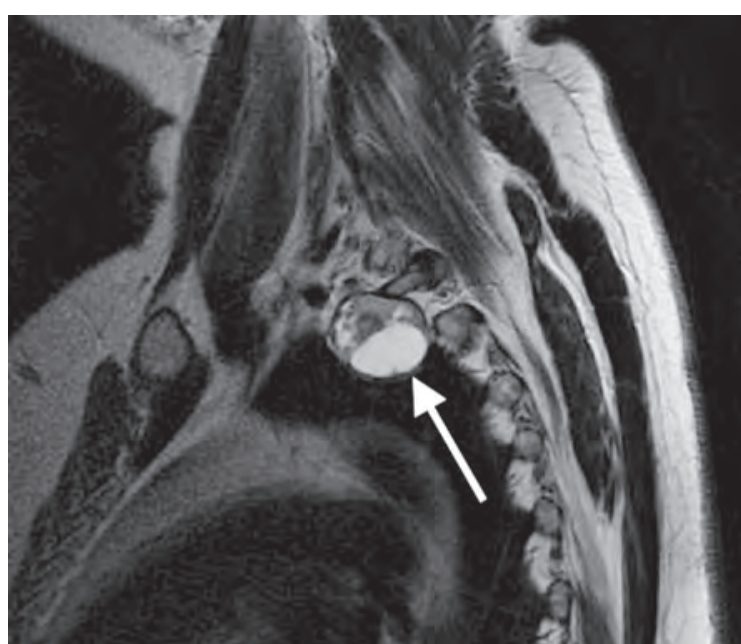

Б (B)
Рис. 2. Магнитно-

резонансная томограмма пациента с апикальной нейрогенной опухолью, проникающей

в межпозвонковое отверстие:

А - фронтальный срез; Б - сагиттальный срез. Опухоль указана стрелкой

Fig. 2. MRI of patient with apical neurogenic tumor (arrow): A - frontal plane; $B$ - axial plane 
Рис. 3. Ламинэктомия: А - вид операционного поля; Б - схема

Fig. 3. Laminectomy: A - operating field view; B - schema

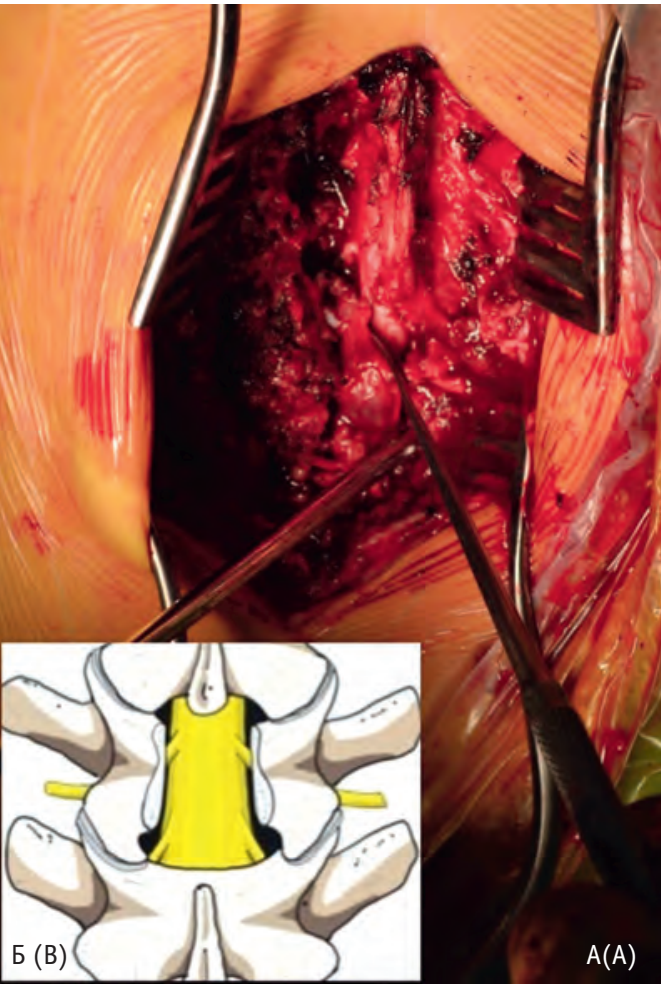

Таблица 3. Хирургические доступы

\begin{tabular}{|l|c|}
\hline \multicolumn{1}{|c|}{ Доступ } & $\boldsymbol{n}(\%)$ \\
\hline ВАТС & $4(28,6)$ \\
\hline Торакотомия + ламинэктомия & $3(21,4)$ \\
\hline Цервикостернотомия + ламинэктомия & $3(21,4)$ \\
\hline ВАТС + ламинэктомия & $2(14,3)$ \\
\hline Надключичный + ламинэктомия & $2(14,3)$ \\
\hline Всего & $14(100)$ \\
\hline
\end{tabular}

BATC - видеоассистированная торакоскопия.

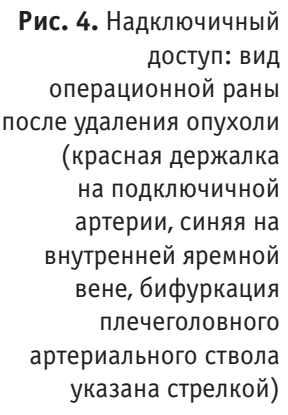

Fig. 4. Supraclavicular approach: view of the surgical wound after removal of the tumor (red holder on the subclavian artery, blue on the internal jugular vein, bifurcation of the brachiocephalic arterial trunk is indicated by an arrow)

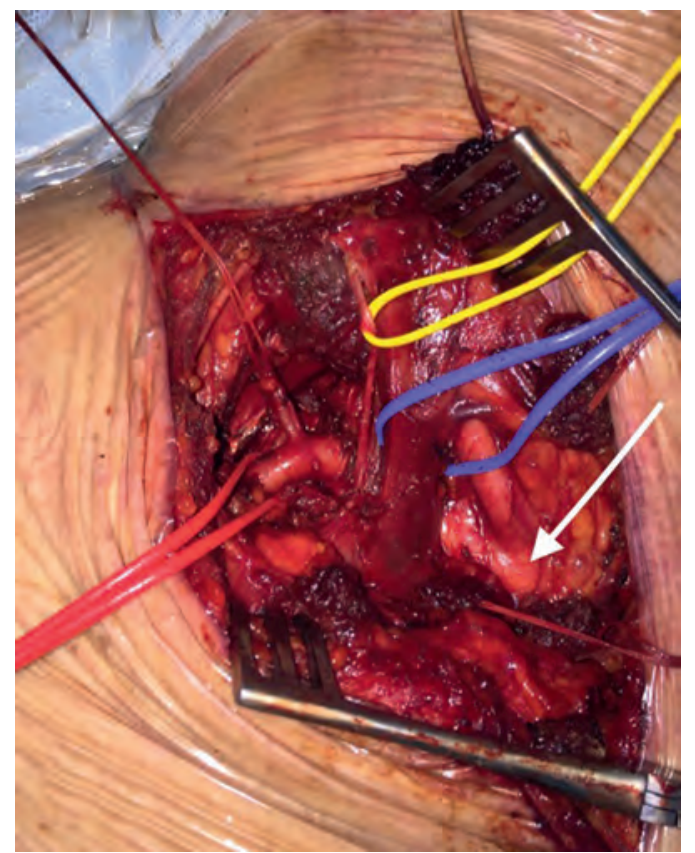

ных опухолей средостения. У 8 (64,3\%) пациентов опухоль была выявлена случайно при прохождении ежегодного профилактического осмотра, а 5 (35,7\%) обратились в связи с болевым синдромом. Клинико-демографические характеристики пациентов с нейрогенными опухолями представлены в табл. 1.

Гистологические типы опухолей представлены в табл. 2.

$8(57,1 \%)$ пациентов имели тип 3 по Eden (IIb по Asazuma). 4 из них опухоль удаляли с применением минимально-инвазивного подхода (у 2 сочетание BATC и гемиламинэктомии, еще у 2 - сочетание надключичного доступа с ламинэктомией). У 6 (46,2\%) был тип 4 по Eden (IIc по Asazuma).

У 4 из них удалось убрать опухоль посредством ВАТС. Хирургические доступы представлены в табл. 3.

Непосредственные результаты операций представлены в табл. 4.

В группе минимально-инвазивных доступов осложнение I степени зарегистрировано у 1 (12,5\%) пациента - апикальный пневмоторакс. В группе стандартных доступов зарегистрированы следующие осложнения: I степени - у 2 (33,3\%) малый гидроторакс и синдром Горнера; II степени - пневмония у 1 (16,7\%); III степени у 2 (33,3\%) ликворея и внутриплевральное кровотечение.

При динамическом наблюдении локального рецидива не выявлено ни у одного пациента. Медиана наблюдения - 68,6 37,2 (от 6 до 124) мес. 1 пациент со злокачественной шванномой умер от прогрессирования опухолевого процесса (отдаленные метастазы) через 24 мес после операции.

\section{Обсуждение}

Первый опыт одноэтапного удаления опухолей типа «гантели» совместной бригадой торакальных хирургов и нейрохирургов был опубликован 0. Akwari и соавт. в 1978 г. [2]. Однако и на сегодняшний день единого подхода нет не только к лечению этих новообразований, но даже к трактовке диагноза. Так, диагноз торакального хирурга «опухоль заднего средостения с распространением в спинномозговой канал» может быть эквивалентен диагнозу нейрохирурга «опухоль спинного мозга типа “песочных часов" или “гантели" с экстравертебральным компонентом».

Хирургическая тактика при таких опухолях сложна и подход должен быть индивидуальным. В литературе описаны различные подходы, включая подходы в соответствии с классификацией Eden и/или Asazuma (Toyama) [3-7]. Однако основные различия сводятся преимущественно к исполь- 
Таблица 4. Непосредственные результаты операций

\begin{tabular}{|l|c|c|c|}
\hline \multicolumn{1}{|c|}{ Показатель } & $\begin{array}{c}\text { Стандартный } \\
\text { доступ }(n=6)\end{array}$ & $\begin{array}{c}\text { Минимально-инвазивный } \\
\text { доступ }(n=8)\end{array}$ & $\begin{array}{c}p \\
\text { Длительность операции, мин }\end{array}$ \\
\hline Интраоперационная кровопотеря, мл & $852,5[202,5 ; 460,0]$ & $192,5[106,2 ; 240,0]$ & 0,053 \\
\hline Длительность дренирования, сут & $7,0[3,2 ; 10,2]$ & $75,0[71,2 ; 97,5]$ & 0,003 \\
\hline Длительность госпитализации, сут & $18,5[10,7 ; 21,2]$ & $1,0[1,0 ; 3,5]$ & 0,029 \\
\hline Необходимость в наркотических анальгетиках, $n(\%)$ & $5(83,3)$ & $5[3,0 ; 7,7]$ & 2,043 \\
\hline Длительность системного приема анальгетиков, сут & $10,5[5,2 ; 14,0]$ & $3,5[2,2 ; 4,7]$ & 0,031 \\
\hline Осложнения, $n(\%)$ & $5(83,3)$ & $1(12,5)$ & 0,013 \\
\hline Летальность, $n(\%)$ & 0 & 0 & 0,008 \\
\hline
\end{tabular}

зованию различных вариантов форамен- или ламинэктомии для мобилизации интраспинального компонента и торакотомии или торакоскопии для мобилизации внутригрудного компонента опухоли. Авторы каждой описываемой методики указывают на какое-либо преимущество: лучшую визуализацию или минимальную травматичность, и практически все исследования основань на изучении небольших групп пациентов. Немаловажное значение имеют установки клиники и предпочтения хирургов. Частота осложнений при хирургическом лечении таких опухолей составляет 30\% [8].

Учитывая травматичность операции, всегда оставалось стремление к минимизации доступа. В 1991 г. H. Osada при опухолях типа «гантели» предложил использовать задний минимально-инвазивный доступ. Однако его применение возможно только при небольшом паравертебральном компоненте [9]. При медиастинальном компоненте больше ширины межреберья, в котором расположена опухоль, необходима резекция задних отделов ребер и/или поперечных отростков позвонков, что лишь увеличивает травматичность. Вариант с кускованием средостенного компонента мы не рассматриваем, так как считаем, что он не соответствует принципам радикализма.

\section{Литература/References}

1. Strollo D.C., Rosado-de-Christenson M.L., Jett J.R. Primary mediastinal tumors: part II. Tumors of the middle and posterior mediastinum. Chest. 1997; 112: 1344-57. DOI: https://doi.org/10.1378/chest.112.5.1344

2. Akwari O.E., Payne W.S., Onofrio B.M., Dines D.E., Muhm J.R. Dumbbell neurogenic tumors of the mediastinum. Diagnosis and management. Mayo Clin Proc. 1978; 53 (6): 353-8.

3. Zairi F., Nzokou A., Sunna T., et al. Minimally invasive costotransversectomy for the resection of large thoracic dumbbell tumors. Br J Neurosurg. 2017;
Первые результаты комбинации ламинэктомии с BATC были опубликованы R. Landreneau и соавт. в 1995 г. [10]. В исследовании X. Chen и соавт. (2019), включавшем 20 пациентов с опухолями по типу «гантели», при использовании ВАТС осложнения были зарегистрированы у 2 пациентов [11]. В этой работе, как и в нашем исследовании, отмечается возможность полностью торакоскопического удаления образования при опухолях, проникающих в межпозвонковое отверстие, но без интраспинального компонента.

Наше исследование ограничено небольшой группой пациентов, что связано с относительной редкостью данной патологии. Точку в данном вопросе могло бы поставить проведение крупных многоцентровых исследований.

\section{Заключение}

Как показало наше исследование, при нейрогенных опухолях по типу «гантели» в качестве хирургического доступа целесообразно сочетать гемиламинэктомию с ВАТС или надключичным доступом (при локализации в верхней апертуре). Эта стратегия характеризуется малой травматичностью, позволяет удалить опухоль в едином блоке и избежать потенциальной опасности рецидива.

31 (2): 179-83. DOI: https://doi.org /10.1080/026886 97.2016 .1233317

4. Asazuma T., Toyama Y., Maruiwa H., Fujimura Y., Hirabayashi K. Surgical strategy for cervical dumbbell tumors based on a three-dimensional classification. Spine (Phila Pa 1976). 2004; 29 (1): E10-4. DOI: https://doi. org /10.1097/01.BRS.0000103662.13689.76

5. Eden K. The dumb-bell tumours of the spine. $\mathrm{Br}$ J Surg. 1941; 28: 549-70.

6. Ando K., Imagama S., Ito Z., et al. Removal of thoracic dumbbell tumors through a single-stage posterior 
approach: its usefulness and limitations. J Orthop Sci. 2013; 18 (3): 380-7. DOI: https://doi.org /10.1007/ s00776-013-0370-9

7. Ando K., Imagama S., Wakao N., et al. Single-stage removal of thoracic dumbbell tumors from a posterior approach only with costotransversectomy. Yonsei Med J. 2012; 53 (3): 611-7. DOI: https://doi.org /10.3349/ ymj.2012.53.3.611

8. Safaee M.M., Lyon R., Barbaro N.M., et al. Neurological outcomes and surgical complications in 221 spinal nerve sheath tumors. J Neurosurg Spine. 2017; 26 (1): 103-11. DOI: https://doi.org /10.3171/2016.5.SPIN E15974
9. Osada H., Aoki H., Yokote K., Taira Y., Yamate N. Dumbbell neurogenic tumor of the mediastinum: a report of three cases undergoing single-staged complete removal without thoracotomy. Jpn J Surg. 1991; 21 (2): 224-8. DOI: https://doi.org/10.1007/BF02470913

10. Vallières E., Findlay J.M., Fraser R.E. Combined microneurosurgical and thoracoscopic removal of neurogenic dumbbell tumors. Ann Thorac Surg. 1995; 59 (2): 469-72. DOI: https://doi.org /10.1016/0003-4975(94)00876-9

11. Chen X., Ma Q., Wang S., Zhang H., Huang D. Surgical treatment of thoracic dumbbell tumors. Eur J Surg Oncol. 2019; 45 (5): 851-6. DOI: https://doi.org / 10.1016/j.ejso.2018.10.536 